\title{
Radioterapia conformada en tres dimensiones con intensidad modulada (IMRT). Nuevas estrategias en tumores de cabeza y cuello
}

\author{
J. Contreras Martínez, I. Herruzo Cabrera
}

\begin{abstract}
Resumen
La radioterapia con intensidad modulada (IMRT) es la forma más sofisticada actualmente disponible para realizar tratamientos radioterápicos con técnicas de planificación conformada en 3 dimensiones (3D-CRT), mediante la utilización de incidencias de campos con intensidad de dosis no uniformes en el volumen blanco. Su utilidad se justifica partiendo de las tres premisas clásicas en radioterapia que nos indican que las dosis más altas de irradiación se traducen en mejor control locorregional de la enfermedad, que el mayor control local se correlaciona con ganancias en supervivencia y que la 3D-CRT nos permitiría aumentar la dosis de irradiación sobre el volumen tumoral con menores efectos secundarios. Se realiza una revisión crítica de las experiencias clínicas actualmente disponibles con IMRT, especialmente enfocada a los tumores de cabeza y cuello, indicando cuales pueden ser las nuevas estrategias de desarrollo en el futuro próximo.
\end{abstract}

Palabras clave:

Radioterapia de intensidad modulada (IMRT). Tumores de cabeza y cuello.

Oncología, 2004; 27 (1):4-12 


\section{Summary}

Intensity-modulated radiotherapy (IMRT) is nowadays the most sophisticated form of radiation therapy to carry out three-dimensional conformal radiotherapy (3D-CRT), employing non-uniform intensities dose beams to cover the target volume. The usefulness is based on three classical radiation therapy hypotheses: higher doses increase the local control of the tumor; a higher local control of the primary tumor improves survival; and three-dimensional conformal radiotherapy allows the delivery of higher radiation doses covering the tumor while minimizing the incidence of secondary effects. A critical review of the publications related with the state of the art of the clinical experience in IMRT, specially those referred to head and neck tumors is made, indicating possible new strategies in the near future.

Key words: Intensity-modulated radiotherapy (IMRD). Head and neck tumors.

\section{Introducción}

Los procesos de planificación y administración de los tratamientos radioterápicos están actualmente en una situación de cambio vertiginoso y radical. Desde los inicios de la radioterapia hasta hoy en día los mayores avances en los tratamientos se han vinculado a una mejor definición del área de irradiación tumoral con lo que se consigue una reducción de la dosis en los tejidos sanos. Este objetivo se empezó a conseguir a mediados de los años 1950 con la introducción en la clínica asistencial de importantes mejoras tecnológicas, como fueron los aparatos de megavoltage (unidades de cobalto ${ }^{60}$ y aceleradores lineales), paralelo al inicio en la utilización de los modernos sistemas de dosimetría clínica y de planificación de tratamientos combinando fotones y electrones de diferentes energías.

Además en las dos últimas décadas se han introducido dos nuevos grupos de avances que nos han permitido dar un paso más en la sofisticación de los tratamientos radioterápicos. En primer lugar la aparición de nuevas técnicas de imagen, que nos permiten delimitar con mayor precisión la localización del tumor (GTV), sus áreas potenciales de expansión de enfermedad subclínica (CTV) y los órganos a riesgo dentro los campos de tratamiento radioterápico, y en segundo lugar el desarrollo de mejores sistemas de planificación de tratamiento en las tres dimensiones del espacio (3D) basados en complejos sistemas informáticos de cálculo, todo ello unido a la disponibilidad de nuevas tecnologías más precisas en la administración de los haces de irradiación. Este conjunto de nuevas aportaciones nos permite, en definitiva, administrar una mayor dosis total de irradiación con una distribución espacial ajustada (conformada) de forma muy precisa al volumen tumoral, respetando los órganos sanos en la vecindad.
El concepto de radioterapia conformada en tres dimensiones (3D-CRT) ha sustituido en muchas situaciones clínicas (tratamientos con intención radical, radioterapia postoperatoria a dosis altas, volúmenes críticos cercanos al tumor, etc.) a las planificaciones realizadas hasta ahora en solo dos dimensiones del espacio (2D). La 3D-CRT conlleva la delimitación de volúmenes tumorales y órganos de riesgo en cada uno de los cortes de la prueba de imagen que se utilice (generalmente $T A C$ ), siendo estos volúmenes necesarios para la planificación del tratamiento radioterápico, realizándose una optimización en la distribución de dosis en el órgano diana mediante un moldeado de los campos de tratamiento (conformación de los campos de tratamiento) al volumen tumoral dibujado en las imágenes de TAC. Esta optimización del tratamiento 3D-CRT realizada se corresponde con la representación de lo que denominamos "beams eye view", que es el campo de tratamiento radioterápico con una conformación determinada, con intensidad de dosis uniforme a través del haz. Dicha intensidad de dosis a su vez puede ser modificada levemente mediante sencillos mecanismos de cuñas o filtros compensadores para una mejor adaptación al volumen blanco diana', 2 .

En Oncología se conoce desde hace mucho tiempo la efectividad de la radioterapia para la erradicación de la mayoría de los tumores humanos. Todo el grupo de avances comentados anteriormente al introducir la 3D-CRT nos permitirán obtener mejores resultados respecto a los tratamientos clásicos en 2D, basándonos en las siguientes premisas clásicamente conocidas en radioterapia ${ }^{3}$ :

1. Dosis más altas de irradiación incrementan el control loco-regional de la enfermedad:

Algunos estudios prospectivos y muchos retrospectivos han demostrado incrementos en el porcentaje de 


\section{J. Contreras Martínez e I. Herruzo Cabrera}

control de la enfermedad con aumentos en la dosis de irradiación en algunas localizaciones tumorales, en particular en los tumores de próstata, cabeza y cuello, pulmón, etc ${ }^{4-10}$.

\section{Un mayor control loco-regional de la enfermedad se} traduce en aumentos de la supervivencia:

Hay abundantes evidencias publicadas (experiencias clínicas y de laboratorio) que demuestran la relación existente entre enfermedad residual (o recidiva) tras tratamiento del tumor primario y las metástasis a distancia, traduciéndose finalmente esto en una disminución de la supervivencia ${ }^{4,5,11,12 . ~}$

\section{La utilización de la 3D-CRT nos permitiría adminis-} trar dosis más altas de irradiación disminuyendo la incidencia de efectos secundarios a largo plazo:

En muchos tipos de tumores el efecto beneficioso de la radioterapia es anulado parcial o totalmente por las complicaciones producidas por la misma en los tejidos sanos, impidiendo completar el esquema terapéutico propuesto. Si se consigue una reducción del volumen en que dichos tejidos no tumorales reciben dosis altas de irradiación se presentarán menores efectos secundarios y podremos mantener el efecto terapéutico ${ }^{13,14}$.

Sin embargo, a pesar de las optimistas experiencias iniciales recogidas con los tratamientos 3D-CRT, se sabe que aproximadamente un $30 \%$ de los pacientes tratados con técnicas convencionales 3D recidivan ${ }^{15}$. Este alto índice de recidivas se justifica por la existencia de subpoblaciones clonógenas tumorales resistentes a las máximas dosis que podemos administrar con los tratamientos radioterápicos convencionales (65-70Gy), debido fundamentalmente a los limites de tolerancia de los tejidos sanos que rodean al volumen blanco tumo- ral'. Los efectos biológicos de la irradiación, tanto a nivel del tumor como de los tejidos sanos, son dosis dependientes, y las curvas correspondientes que describen la relación dosis-respuesta se representan en un modelo teórico con formas sigmoidales en ambos casos, sin embargo el rango de tolerancia al tratamiento es menor para el tejido tumoral. El modelo teórico ideal asume que las curvas de control tumoral y de daño al tejido sano son paralelas en su forma sigmoide y separadas entre si de forma suficiente para poder obtenerse con el tratamiento la curación (Fig. 1.A). Este es el principio en el que se basa la curación con el tratamiento radioterápico. Pero la realidad es otra, y los datos clínicos nos muestran que la inclinación o pendiente de la curva del control tumoral es siempre menor que la de los efectos secundarios y esto nos limita la capacidad de administrar las dosis altas de irradiación necesarias para controlar el tumor ${ }^{15,16}$ (Fig. 1.B).

Con los tratamientos 3D-CRT en muchas ocasiones se aprecia que el grado de conformación de la dosis al volumen diana tumoral y la capacidad de excluir los órganos de riesgo no es la óptima, salvo en casos de tumores pequeños y con formas relativamente sencillas, pero que además requieren ser tratados con múltiples campos ${ }^{17}$. Por ello, e incluso antes de que esta forma de tratamiento con radioterapia conformada en 3D (que denominaremos 3D-CRT clásica) haya sido completamente introducida de forma generalizada en los servicios de Oncología Radioterápica, empieza a surgir un nuevo tipo de planificación y administración de tratamiento con radioterapia conformada, la denominada radioterapia de intensidad modulada (IMRT).

La IMRT es una nueva forma de 3D-CRT basada en el uso de haces de irradiación optimizados, además, mediante incidencias de campos con intensidad de dosis no

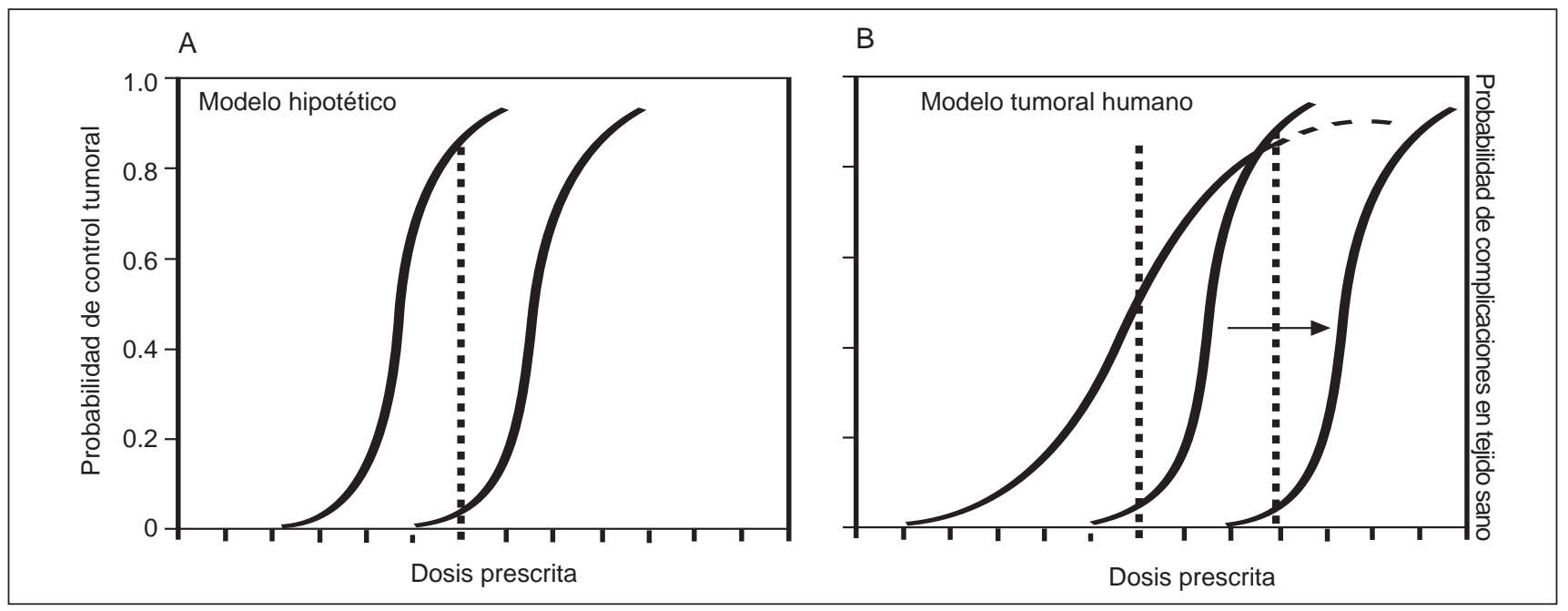

Figs. $1 \mathrm{~A}$ y $1 \mathrm{~B}$ 
uniformes adaptadas al volumen blanco ${ }^{18}$. La IMRT representa un avance muy reciente, que inicia su desarrollo a finales de la década de los años ochenta ${ }^{19}$, estando actualmente en una fase inicial de implantación dentro de los servicios de Oncología Radioterápica, que nos brinda la oportunidad de administrar dosis más altas de irradiación que las clásicamente empleadas por la posibilidad de conseguir una mayor precisión en la distribución conformada en el tumor ${ }^{18,20,21}$. Con IMRT podemos conseguir plantear casi cualquier distribución de la dosis de irradiación con una abrupta caída de la misma en los límites entre el volumen del tumor y los tejidos sanos de la vecindad, reduciéndose en dichos teiidos tanto la dosis final como el volumen que la recibe. Por tanto la ventaja fundamental que nos aporta la 3D-CRT en su modalidad de IMRT es la reducción del volumen de tejido sano expuesto a dosis altas de irradiación, lo cual nos permite conseguir una escalada de dosis a nivel del tumor.

Diferentes tejidos sanos (mucosa orofaríngea, tracto gastrointestinal, pulmón, hígado, etc.) muestran una estrecha relación directa entre el volumen irradiado y la intensidad del daño tisular o de efectos secundarios ${ }^{22}$. Por consiguiente, si disminuimos el volumen global de irradiación desplazaremos hacia la derecha (hacia la zona de mayor dosis) la curva de complicaciones achacables al tratamiento en tejidos sanos, permitiendo esto realizar una escalada de dosis en el tumor antes de alcanzarse los límites de complicaciones aceptables. Algunas experiencias clínicas en cáncer de próstata confirman lo afirmado anteriormente, por ejemplo en pacientes tratados con técnicas 3D-CRT hasta 70.2 y 75.6Gy sobre el PTV, la dosis media apreciada en los histogramas dosis-volumen en recto eran superiores en los pacientes que presentaban sangrado rectal como complicación frente aquellos en los que no lo presentaban ${ }^{23}$.

Hasta ahora la IMRT ha sido probada clínicamente en numerosos centros distribuidos por todo el mundo, y las múltiples experiencias publicadas reflejan una mejoría en cuanto a la precisión en la distribución de la dosis en el volumen diana tumoral para determinadas localizaciones, como por ejemplo en el cáncer de próstata, donde se aprecia una reducción de la toxicidad rectal que permite una escalada de dosis hasta niveles hasta ahora inalcanzables (82-90Gy) $)^{6,24-31}$. También existen experiencias en tumores de la región de cabeza y cuello7, 17, $24,32-36$, tiroides ${ }^{37}$, sistema nervioso central ${ }^{24}$, mama ${ }^{38-40}$, ginecológicos, etc.

\section{Planificación y administración de IMRT}

Como se ha mencionada anteriormente, la IMRT es una forma más sofisticada de tratamiento 3D-CRT, utilizando unos sistemas específicos de administración de irradiación que consiguen "conformar" de forma extremadamente precisa la distribución de la dosis sobre el volumen blanco tumoral. El especialista debe definir (pintar) los contornos anatómicos de cada una de las localizaciones (tumor primario, áreas de enfermedad microscópica, órganos de riesgo), especificar las dosis total y por sesión que se quiere administrar en cada uno de volúmenes blancos, y aceptar o no (es una decisión clínica distinta en cada caso) el grado de inhomogeneidad de dosis existente dentro del volumen tumoral. Para ello previamente se tiene que introducir en los sistemas de planificación específicos para IMRT unas condiciones de "restricción" de dosis (constrains) teniendo en cuenta las diferentes tolerancias de cada uno de los órganos de riesgo, y la dosis máxima y mínima que se consideran aceptables en cada PTV.

Al contrario de que ocurre con los sistemas de planificación convencionales en los que se emplea una selección arbitraria de especificaciones de los campos de tratamiento (ángulos, tamaños, formas, cuñas, pesos, etc.) que se van ajustando mediante el método de ensayo y error hasta que se consigue una distribución de dosis satisfactoria, en los tratamientos IMRT se utilizan unos sistemas de planificación inversa con optimizaciones informatizadas, que generan unos campos de tratamientos con perfiles de intensidad variable lo más ajustado posible a los criterios predefinidos por el especialista respecto a la distribución de dosis en el tumor y los órganos de riesgo circundantes (constrains), con unas rangos de posible violación de la dosis prescritas (penalties) especificadas para cada tipo de tumor y órganos de riesgo en función de la situación clínica de cada paciente ${ }^{44}$.

La técnica IMRT esta actualmente en un proceso de cambio y desarrollo constante, existiendo por tanto muchos sistemas diferentes para la planificación y administración de los tratamientos. En general se requiere de aceleradores lineales con sistemas multiláminas, tanto en su forma dinámica como en la modalidad de multisegmentos estáticos (step-and-shoot), o también con sistemas de tomoterapia ${ }^{19}, 24,25$. Lo que se genera mediante la planificación inversa de los tratamientos IMRT son una serie de campos con perfiles de intensidad variable que se usan como plantilla de referencia para la administración del tratamiento. La apertura de los campos viene determinada por un patrón de intensidad generado en el proceso de optimización de la planificación, y, por ejemplo, con el desplazamiento de las hojas del sistema multiláminas dinámicas se genera una "ventana deslizante" (sliding window) con una forma y velocidad que se modifica a través de la amplitud del campo de tratamiento de forma dinámi$\mathrm{ca}$, ajustándose constantemente al perfil de intensidad prescrito mediante un control informático. 


\section{J. Contreras Martínez e I. Herruzo Cabrera}

\section{IMRT en tumores de cabeza y cuello}

Existen muchos trabajos describiendo las experiencias de diferentes centros en cuanto a planificación, administración y control de calidad con tratamientos IMRT, la mayoría de ellos recogidos en una puesta al día publicada en el 2001 por el Grupo cooperativo para estudio de la radioterapia de intensidad modula$\mathrm{da}^{18}$. Sin embargo, existen escasos datos clínicos en la literatura científica que nos presenten resultados que verifique el beneficio potencial de esta técnica y la ausencia de riesgos a largo plazo, siendo realizados la mayoría de los trabajos en pacientes con tumores de próstata $^{6,24-31}$.

La región de cabeza y cuello es una localización atractiva para el desarrollo de la IMRT, dado que se trata de un área donde la motilidad de los órganos internos es mínima, existen en la proximidad de los volúmenes dianas órganos de riesgo críticos considerados como muy radiosensibles (parótida, troncoencéfalo, canal medular, etc.), y además disponemos de sistemas fiables de inmovilización para poder reproducir diariamente los campos de tratamientos ${ }^{46}$. La posibilidad que nos ofrece la IMRT de conseguir gradientes muy precisos de dosis ajustados a los órganos diana, limitando la dosis que reciben los tejidos sanos de alrededor, nos permite incrementar el beneficio terapéutico a la vez que reducir los efectos secundarios en los tejidos sanos de la vecindad (glándulas salivares mayores y menores, mandíbula, musculatura parafaringea, y en tumores nasofaringeos o de senos paranasales el oído interno, articulación temporomandibular, lóbulos temporales y vía óptica). Además, la IMRT elimina la necesidad de utilizar campos con electrones para cubrir las cadenas cervicales posteriores, clásicamente empleados en los tratamientos convencionales y que se suelen asociar con deficiencias disimétricas y dificultades para su aplicación.

Otra de las ventajas que aportan los tratamientos IMRT es que generalmente, como se comentó en la introducción, se administra solo un plan de campos durante todo el curso del tratamiento, consiguiendo una mejor conformación de la dosis en el volumen blanco comparado con los planes de reducciones consecutivas de campos de tratamiento (campos iniciales, exclusión medular y sobreimpresión). Cuando se realiza un plan único de tratamiento los GTVs reciben más dosis total final y mayor dosis por fracción que la que reciben los CTVs y los órganos de riesgo, traduciéndose esto incluso en lo que se denomina una mayor dosis equivalente biológica (BED) a nivel de los GTVs. Este tipo de distribución de dosis variables dentro de las áreas de tratamiento es lo que se viene a denominar como "bo- ost simultáneo integrado" (simultaneous integrated boost o SIB) 33,34 .

Otra de las razones por las que es interesante el desarrollo de la IMRT en los tumores de cabeza y cuello es la existencia de datos clínicos que nos indican de forma clara la relación directa entre la dosis de radioterapia que se administra, el control locorregional de la enfermedad y la supervivencia-metástasis a distan$\mathrm{cia}^{11}{ }^{17}$. Sin embargo para conseguir estos objetivos debemos tener en cuenta diferentes e importantes factores que se implican de forma entrelazada. En primer lugar se requiere de una precisa delimitación del volumen microscópico tumoral (GTV) y de los volúmenes que contienen la enfermedad microscópica potencialmente asociada (CTV). Sin embargo, en la mayoría de los casos, el TAC de diagnóstico junto al de simulación (a veces sin contraste) es la única modalidad de imagen que se dispone para delimitar dichos volúmenes en los tumores de cabeza y cuello. La RNM es necesaria (junto al TAC) en los tumores cercanos a la base del cráneo (por ejemplo tumores de nasofaringe o senos paranasales), pues nos da mayor información de la que nos aporta exclusivamente el TAC respecto a la extensión tumoral en esas zonas y la posible afectación de los espacios para y retrofaringeos ${ }^{48}$.

Por tanto hay que tener en cuenta que una de las mayores potenciales "trampas" en la utilización de la IMRT sería el error al seleccionar y delimitar de forma excesivamente precisa o limitada el CTV. Para la correcta delimitación del CTV se requiere de un conocimiento exhaustivo de la anatomía de la región de cabeza y cuello y su correlación con las correspondientes pruebas de imágenes, así como el conocimiento de los patrones de extensión locorregional de la enfermedad subclínica que se debe incluir dentro de los campos de tratamiento, teniendo en cuenta el porcentaje de riesgo de afectación en cada uno de los niveles cervicales según el origen del tumor, como bien reflejan Gregoire y Novak en sus publicaciones ${ }^{49,50}$

En estos momentos se están probando en tumores de cabeza y cuello múltiples esquemas distintos de fraccionamientos y de dosis total a administrar teniendo en cuenta diferentes consideraciones radiobiológicas para su diseño, estando aún pendiente de evaluar los resultados ${ }^{44}$. Sin embargo, con fines puramente didácticos para la redacción y entendimiento de este artículo, se podrían hacer dos grupos genéricos de fraccionamientos en los que agrupar las diferentes formas de aproximación radiobiológica del tratamiento IMRT en tumores de cabeza y cuello:

- En un primer grupo se administra al GTV dosis con fraccionamiento estándar (por ejemplo 70Gy, en 35 sesiones de 2.0Gy). En este planteamiento los volúmenes 
dianas seleccionados reciben dosis bajas totales y por sesión (63Gy a las zonas de mayor riesgo o enfermedad macroscópica y 58.1Gy a los CTVs de menor riesgo, con una dosis por cada una de las 35 sesiones de 1.8 y 1.66Gy respectivamente). Estos esquemas de tratamientos se utilizan generalmente en protocolos donde los tumores están en estadios avanzados, se prevé administrar tratamiento concomitante con quimioterapia, con volúmenes blancos amplios, y que se espera por tanto que se produzca mayor toxicidad.

- La segunda estrategia de tratamiento lo que hace es acomodar las diferencias en la dosis por fracción a cada volumen, administrando una dosis por fracción superior a la estándar en el GTV y manteniendo la dosis por fracción estándar en el CTV. Esta estrategia es la que ha adoptado el Radiation Therapy Oncology Group (RTOG) para el estudio de la IMRT en el cáncer de orofaringe $(\mathrm{H}$ 0022). En este estudio el GTV recibe una dosis total de 66Gy en 30 fracciones a 2.2Gy/fracción. Los CTVs de mayor riesgo reciben $60 \mathrm{~Gy}$ y los de bajo riesgo $54 \mathrm{~Gy}$, a 2.0 y $1.8 \mathrm{G}$ y respectivamente. Esto se traduce en una dosis nominal total en el GTV de 70Gy en 6 semanas (esquema similar en tiempo y dosis final al que se administra con curso de tratamiento convencional acelerado) ${ }^{51}$.

Respecto a otras experiencias clínicas con IMRT en tumores de cabeza y cuello se puede destacar la publicada por Lee y cols, en la que refieren una supervivencia libre de enfermedad del $97 \%$ a los 31 meses en una amplia serie de pacientes con tumores de nasofaringe, lo cual es un punto de inflexión en cuanto a resultados de supervivencia en el tratamiento de los tumores de cabeza y cuello ${ }^{52}$. Además no presentan una aparente mayor toxicidad aguda comparada con la clásicamente recogida con los tratamientos de radioterapia convencional y quimioterapia, aunque habrá que esperar un periodo de seguimiento mas largo para establecer si se mantiene la ausencia de toxicidad crónica. Al analizar los resultados publicados por Lee y cols vemos que en su serie se realiza una muy precisa delimitación del GTV y CTV, utilizando en todos los casos TAC de simulación con contraste y RNM, superponiendo las imágenes obtenidas con ambas técnicas. Otro de los factores que puede haber influido en sus resultados positivos seria la administración de mayores dosis total y por fracción al volumen blanco, y la disminución de la dosis recibida en los órganos de riesgo. En su serie la dosis prescrita es de 70Gy al GTV con un fraccionamiento de 2.25Gy/fracción, 3133 fracciones. Teniendo en cuenta las inhomogeneidades de dosis en los volúmenes blancos y para evitar subdosificaciones la dosis media en el GTV fue de 74.5Gy (con 2.24-2.4Gy por fracción), que traducido a dosis total normalizada (NTD) equivale a $80 \mathrm{~Gy}$. Estas dosis medias administradas son similares a las utilizadas por $\mathrm{Bu}$ - tler y cols. ${ }^{33}$, cuyo esquema consiste en prescribir 60Gy en 25 fracciones, con una dosis media de 64.5Gy a 2.6Gy/fracción sobre el GTV, que traducido a NTD supone $72 \mathrm{~Gy}$ administrados en 5 semanas, presentando aceptables efectos secundarios agudos pero pendiente de establecer las complicaciones a largo plazo así como la supervivencia.

Finalmente se ha de destacar la existencia de múltiples experiencias en las que se describe una disminución en la dosis de irradiación que reciben las glándulas parótidas y otros órganos de riesgo en tumores nasofaringeos cuando se utiliza IMRT frente a tratamientos convencionales, con menores efectos secundarios agudos durante el tratamiento, sin embargo (aunque parece probable) aún no esta claramente establecida una disminución clínica de los efectos secundarios a largo plazo $32,35,52-55$. En un trabajo realizado por Hunt y cols. en el Memorial Sloan Kettering Cancer Center de Nueva York ${ }^{32}$ en el que comparan los resultados de distribución de dosis en 6 pacientes en los que se realizan las planificaciones de forma convencional, en 3D-CRT y con IMRT, aprecian una mejor cobertura de la dosis al PTV con IMRT (fundamentalmente en región parafaringea, base de cráneo y zona medial de las cadenas ganglionares) frente a las técnicas convencional y con 3D-CRT, con una disminución de la dosis recibida en mandíbula, lóbulos temporales, médula y parótidas que oscilaba entre 10-15\% de forma general, sin embargo esta disminución de dosis no fue suficiente para preservar la función salivar de forma aguda. Como resultados preliminares también refieren un mayor control local del tumor con IMRT frente a los otros tratamientos convencionales, estando pendientes de presentar un seguimiento a más largo plazo.

\section{Reflexiones finales. Líneas de futuro}

En radioterapia, como en cualquier otro campo de la medicina, cada avance técnico que se produce soluciona determinados problemas generando a su vez otros nuevos. Se ha de reconocer que la IMRT abre una nueva era dentro de la Oncología Radioterápica, permitiéndonos la posibilidad, siempre perseguida en Radioterapia, de ajustarse lo máximo posible a los volúmenes blancos diana respetando los órganos de riesgo de la vecindad, consiguiendo una precisión que hasta ahora no había sido posible. Los datos preliminares nos indican que con IMRT podemos mejorar los resultados en cuanto al control local en tumores de próstata y la región de cabeza y cuello (fundamentalmente naso y orofaringe), aunque probablemente se demuestre beneficio en un futuro en otras localizaciones tumorales. Además la IMRT nos ofrece en algunos casos la posibilidad de reducir la toxici- 


\section{J. Contreras Martínez e I. Herruzo Cabrera}

dad derivada de los efectos secundarios con el tratamiento.

Los tumores de cabeza y cuello representan dentro de la Oncología una región atractiva para el desarrollo de la IMRT debido a la conocida relación existente en esta localización entre dosis de irradiación-control locorregional de la enfermedad-supervivencia, además la motilidad de los órganos internos en esta área es mínima, existiendo en la proximidad de los volúmenes dianas órganos de riesgo críticos que se consideran muy radiosensibles (parótida, troncoencéfalo, canal medular, etc.), y se dispone de sistemas fiables de inmovilización para poder reproducir diariamente los campos de tratamientos. Otra ventaja con IMRT seria la posibilidad de eliminar la necesidad de utilizar campos con electrones en las cadenas cervicales posteriores, clásicamente empleados en los tratamientos convencionales y que se suelen asociar con deficiencias dosimétricas y dificultades para su reproducibilidad diaria.

Sin embargo la tecnología que disponemos hoy en día para administrar tratamientos IMRT de alta precisión es superior a la capacidad de las actuales técnicas de imagen para precisar cuales son los límites del tumor frente a los tejidos sanos. Hoy por hoy la IMRT es una técnica compleja, costosa, que requiere una gran dedicación (de tiempo y recursos) y que tiende a producir mayor número de errores, averías y dificultad en el control de calidad de los tratamientos frente a los tratamientos convencionales. Además conlleva una mayor exposición corporal a dosis bajas de irradiación de consecuencias a largo plazo que en este momento no son conocidas.

Por tanto para conseguir en un futuro próximo una mayor implementación de la IMRT dentro de la Oncología Radioterápica se deberían realizar progresos en diferentes direcciones:

- Disponibilidad de una mayor precisión en la delimitación de los volúmenes diana, tanto de enfermedad macroscópica como subclínica. Para ello se deben conocer y tener en cuenta las características clínicas y biológicas específicas de cada tumor, sus vías de diseminación, las áreas ganglionares de riesgo, etc. Esto se conseguirá incorporando las nuevas técnicas de imagen biológicas y moleculares (PET, RNM espectroscópica, SPECT...) que nos aportan una información más "fisiológica y funcional" del comportamiento de los tumores, así como con la adquisición de modernos simuladores PET-TAC que nos permitan integrar las imágenes funcionales con las anatómicas de la RNM.

- Desarrollo de una nueva generación de sistemas de inmovilización y sistemas de adquisición de imágenes de simulación en los que se tengan en cuenta los movimientos de órganos en la planificación del tratamiento.
- Nuevas técnicas de optimización en los sistemas de planificación para integración de los márgenes específicos de PTV según las imágenes adquiridas en la simulación específicamente para cada tipo de tumor en un determinado paciente.

- Disponibilidad de nuevas técnicas de imagen para verificación y control de calidad durante los tratamientos, etc.

En definitiva la IMRT nos ofrece una oportunidad única de introducir en la radioterapia clínica los conocimientos radiobiológicos, con la posibilidad de modular en diferentes tejidos las dosis total y por sesión administradas, en función de la gran variabilidad (en cuanto agresividad y radiosensibilidad) que presentan los tumores dentro de una misma localización o una similar histología, posibilidad de adaptación de los tratamientos a la situación clínica especifica de cada paciente, como por ejemplo en caso de necesidad de utilización de quimioterapia simultanea a la radioterapia (con mayor toxicidad), estado general del paciente, etc. Por tanto con la IMRT tendremos que abandonar el concepto clásico de dosis de radioterapia estándar, y diseñar tratamientos individualizados (a la carta) para un determinado paciente, con un tumor específico, dependiendo de su comportamiento biológico y de los factores pronósticos de agresividad local o sistémica (hipoxia, índice proliferativo, etc.), que en un futuro próximo nos aportarán las nuevas pruebas de imágenes funcionales.

En estos momentos la IMRT es una técnica que esta en la fase inicial de su desarrollo, sin unos resultados clínicos positivos claramente establecidos, y que no muchos servicios de Oncología Radioterápica tienen incorporada, sin embargo adoptar una política de "esperar y ver" podría ser un error en centros considerados avanzados para el tratamiento oncológico, pues aunque la IMRT no se puede considerar en si misma como un fin, si es un medio que se puede utilizar para conseguir el desarrollo de la radioterapia conformada en 3D más sofisticada que hoy disponemos. Mientras tanto esperemos que en los próximos años los resultados de los trabajos de investigación sean los que nos contesten en que situaciones clínicas la IMRT aportará alguna ventaja en el tratamiento del cáncer respecto a la radioterapia clásica.

\author{
Correspondencia: \\ Dr. Jorge Contreras Martínez \\ Servicio de Oncología Radioterápica \\ Complejo Hospitalario Carlos Haya \\ Avenida Carlos Haya S/N \\ E-29010 Malaga \\ E-mail: jorge.contreras.sspa@juntadeandalucia.es \\ contrerasjorge@hotmail.com
}




\section{Bibliografía}

1. Leibel SA, Ling CC, Kutcher GJ, Mohan R, Cordon-Cordo C, Fuks Z. The biological basis for conformal three-dimensional radiation therapy. Int J Radiat Oncol Biol Phys 1991;21(3):805-11.

2. Fuks Z, Horwich A. Clinical and technical aspects of conformal therapy. Radiat Oncol 1993;29:219-220.

3. Zapatero A. 3D conformal radiotherapy and intensity-modulated radiotherapy: towards dose optimization. Rev Oncol 2002;4(2):103-7.

4. Tubiana $M$. The role of local treatment in the cure of cancer. Eur J Cancer 1992;28A(12):2061-9.

5. Suit HD. Local control and patient survival. Int J Radiat Oncol Biol Phys 1992;23(3):653-60.

6. Zelefsky M, Fuks Z, Hunt M, Lee HJ, Lombardi D, Ling CC et al. High dose radiation delivered by intensity modulated conformal radiotherapy improves the outcome of localized prostate cancer. J Urol 2001;166(3):876-81.

7. Chao KS, Low DA, Perez CA, Purdy JA. Intensity-modulated radiation therapy in head and neck cancers: The Mallinckrodt experience. Int J Cancer 2000;90(2):92-103.

8. Eisbruch A. Head and neck cancer: advances in radiation treatment. MedGenMed 2002;4(3):16.

9. Eisbruch A. Intensity-modulated radiotherapy of head-andneck cancer: encouraging early results. Int J Radiat Oncol Biol Phys 2002;53(1):1-3.

10. Hanks GE, Hanlon AL, Schultheiss TE, Pinover WH, Movsas $B$, Epstein BE, et al. Dose escalation with $3 D$ conformal treatment: five year outcomes, treatment optimization, and future directions. Int J Radiat Oncol Biol Phys 1998;41(3):501-10.

11. Leibel SA, Scott CB, Mohiuddin M, Marcial VA, Coia LR, Davis LW, et al. The effect of local-regional control on distant metastatic dissemination in carcinoma of the head and neck: results of an analysis from the RTOG head and neck database. Int J Radiat Oncol Biol Phys 1991;21(3):54956.

12. Fuks Z, Leibel SA, Wallner KE, Begg CB, Fair WR, Anderson $\mathrm{LL}$, et al. The effect of local control on metastatic dissemination in carcinoma of the prostate: long-term results in patients treated with $125 \mathrm{I}$ implantation. Int J Radiat Oncol Biol Phys 1991;21(3):537-47.

13. Martel MK, Ten Haken RK, Hazuka MB, Turrisi AT, Fraass BA, Lichter AS. Dose-volume histogram and 3-D treatment planning evaluation of patients with pneumonitis. Int $\mathrm{J} \mathrm{Ra-}$ diat Oncol Biol Phys 1994;28(3):575-81.

14. Oetzel D, Schraube P, Hensley F, Sroka-Perez G, Menke $M$, Flentje M. Estimation of pneumonitis risk in three-dimensional treatment planning using dose-volume histogram analysis. Int J Radiat Oncol Biol Phys 1995;33(2):455-60.

15. Thames H, Schultheiss T, Hendry J. Can modest escalations of dose be detected as increased tumor control. Int J Radiat Oncol Biol Phys 1994;22:241-246.
16. Bentzen SM. Radiobiological considerations in the design of clinical trials. Radiother Oncol 1994;32(1):1-11.

17. Verhey $\amalg$. Comparison of three-dimensional conformal radiation therapy and intensity-modulated radiation therapy systems. Semin Radiat Oncol 1999;9(1):78-98.

18. Intensity-modulated radiotherapy: current status and issues of interest. Int J Radiat Oncol Biol Phys 2001;51(4):880914.

19. Brahme A. Optimization of stationary and moving beam radiation therapy techniques. Radiother Oncol 1988; 12(2):129-40.

20. Tubiana M, Eschwege F. Conformal radiotherapy and intensity-modulated radiotherapy--clinical data. Acta Oncol 2000;39(5):555-67.

21. Leibel SA, Fuks Z, Zelefsky MJ, Wolden SL, Rosenzweig $\mathrm{KE}$, Alektiar KM, et al. Intensity-modulated radiotherapy. Cancer J 2002;8(2):164-76.

22. Withers HR, Taylor JM, Maciejewski B. Treatment volume and tissue tolerance. Int J Radiat Oncol Biol Phys 1988;14(4):751-9.

23. Jackson A, Skwarchuk MW, Zelefsky MJ, Cowen DM Venkatraman ES, Levegrun S, et al. Late rectal bleeding after conformal radiotherapy of prostate cancer. II. Volume effects and dose-volume histograms. Int J Radiat Oncol Biol Phys 2001;49(3):685-98.

24. Fraass BA, Kessler ML, McShan DL, Marsh LH, Watson BA, Dusseau WJ, et al. Optimization and clinical use of multisegment intensity-modulated radiation therapy for high-dose conformal therapy. Semin Radiat Oncol 1999;9(1):60-77.

25. Zelefsky MJ, Fuks Z, Happersett L, Lee HJ, Ling CC, Burman $C M$, et al. Clinical experience with intensity modulated radiation therapy (IMRT) in prostate cancer. Radiother Oncol 2000;55(3):241-9.

26. Pollack A, Zagars GK, Rosen, II. Prostate cancer treatment with radiotherapy: maturing methods that minimize morbidity. Semin Oncol 1999;26(2):150-61.

27. Nutting CM, Convery DJ, Cosgrove VP, Rowbottom C, Padhani AR, Webb $S$, et al. Reduction of small and large bowel irradiation using an optimized intensity-modulated pelvic radiotherapy technique in patients with prostate cancer. Int J Radiat Oncol Biol Phys 2000;48(3):649-56.

28. Teh BS, Mai WY, Uhl BM, Augspurger ME, Grant WH, 3rd, Lu HH, et al. Intensity-modulated radiation therapy (IMRT) for prostate cancer with the use of a rectal balloon for prostate immobilization: acute toxicity and dose-volume analysis. Int J Radiat Oncol Biol Phys 2001 ;49(3):705-12.

29. Xia P, Pickett B, Vigneault E, Verhey $U$, Roach M, 3rd. Forward or inversely planned segmental multileaf collimator IMRT and sequential tomotherapy to treat multiple dominant intraprostatic lesions of prostate cancer to $90 \mathrm{~Gy}$. Int J Radiat Oncol Biol Phys 2001;51(1):244-54.

30. Shu HK, Lee T, Vigneauly E, Xia P, Pickett B, Phillips TL, et 


\section{J. Contreras Martínez e I. Herruzo Cabrera}

al. Toxicity following high-dose three-dimensional conformal and intensity-modulated radiation therapy for clinically localized prostate cancer. Urology 2001;57(1):102-7.

31. Klein EE, Low DA, Sohn JW, Purdy JA. Differential dosing of prostate and seminal vesicles using dynamic multileaf $\mathrm{co}^{-}$ llimation. Int J Radiat Oncol Biol Phys 2000;48(5):1447-56.

32. Hunt MA, Zelefsky M, Wolden S, Chui CS, LoSasso T, Rosenzweig $\mathrm{K}$, et al. Treatment planning and delivery of intensity-modulated radiation therapy for primary nasopharynx cancer. Int J Radiat Oncol Biol Phys 2001;49(3):623-32.

33. Butler EB, Teh BS, Grant WH, 3rd, Uhl BM, Kuppersmith RB, Chiu JK, et al. Smart (simultaneous modulated accelerated radiation therapy) boost: a new accelerated fractionation schedule for the treatment of head and neck cancer with intensity modulated radiotherapy. Int J Radiat Oncol Biol Phys 1999;45(1):21-32.

34. Chao KS, Deasy JO, Markman J, Haynie J, Perez CA Purdy JA, et al. A prospective study of salivary function sparing in patients with head-and-neck cancers receiving intensity-modulated or three-dimensional radiation therapy: initial results. Int J Radiat Oncol Biol Phys 2001;49(4):907-16

35. Cheng JC, Chao KS, Low D. Comparison of intensity modulated radiation therapy (IMRT) treatment techniques for nasopharyngeal carcinoma. Int J Cancer $2001 ; 96(2): 126-31$.

36. Dawson LA, Anzai Y, Marsh L, Martel MK, Paulino A, Ship $J A$, et al. Patterns of local-regional recurrence following parotid-sparing conformal and segmental intensity-modulated radiotherapy for head and neck cancer. Int J Radiat Oncol Biol Phys 2000;46(5):1 11 17-26.

37. Nutting CM, Convery DJ, Cosgrove VP, Rowbottom C, Vini $L$, Harmer $C$, et al. Improvements in target coverage and reduced spinal cord irradiation using intensity-modulated radiotherapy (IMRT) in patients with carcinoma of the thyroid gland. Radiother Oncol 2001;60(2):173-80.

38. Hong L, Hunt M, Chui C, Spirou S, Forster K, Lee $H$, et al. Intensity-modulated tangential beam irradiation of the intact breast. Int J Radiat Oncol Biol Phys 1999;44(5): 11 155-64.

39. Kestin LL, Sharpe MB, Frazier RC, Vicini FA, Yan D, Matter $R C$, et al. Intensity modulation to improve dose uniformity with tangential breast radiotherapy: initial clinical experience. Int J Radiat Oncol Biol Phys 2000;48(5):1559-68.

40. van Asselen B, Raaijmakers CP, Hofman P, Lagendijk JJ. An improved breast irradiation technique using three-dimensional geometrical information and intensity modulation. Radiother Oncol 2001;58(3):341-7.

41. Hong L, Alektiar K, Chui C, LoSasso T, Hunt M, Spirou S, et al. IMRT of large fields: whole-abdomen irradiation. Int J Radiat Oncol Biol Phys 2002;54(1):278-89.

42. Roeske JC, Lujan A, Rotmensch J, Waggoner SE, Yamada D, Mundt AJ. Intensity-modulated whole pelvic radiation therapy in patients with gynecologic malignancies. Int J Radiat Oncol Biol Phys 2000;48(5):1613-21.
43. Portelance L, Chao KS, Grigsby PW, Bennet H, Low D. Intensity-modulated radiation therapy (IMRT) reduces small bowel, rectum, and bladder doses in patients with cervical cancer receiving pelvic and para-aortic irradiation. Int $J$ Radiat Oncol Biol Phys 2001;51(1):261-6.

44. Mohan R, Wu Q, Manning M, Schmidt-Ullrich R. Radiobiological considerations in the design of fractionation strategies for intensity-modulated radiation therapy of head and neck cancers. Int J Radiat Oncol Biol Phys 2000;46(3):619-30.

45. Brahme A. Optimization of radiation therapy. Int J Radiat Oncol Biol Phys 1994;24:158.

46. Tsai JS, Engler MJ, Ling MN, Wu JK, Kramer B, Dipetrillo $\mathrm{T}$, et al. A non-invasive immobilization system and related quality assurance for dynamic intensity modulated radiation therapy of intracranial and head and neck disease. Int J Radiat Oncol Biol Phys 1999;43(2):455-67.

47. Anderson P, Dische S. Local tumor control and the subsequent incidence of distant metastatic disease. Int J Radiat Oncol Biol Phys 1981;7(12):1645-8.

48. Som PM. The present controversy over the imaging method of choice for evaluating the soft tissues of the neck. AJNR Am J Neuroradiol 1997;18(10):1869-72.

49. Gregoire V, Coche E, Cosnard G, Hamoir M, Reychler H. Selection and delineation of lymph node target volumes in head and neck conformal radiotherapy. Proposal for standardizing terminology and procedure based on the surgical experience. Radiother Oncol 2000;56(2):135-50.

50. Nowak PJ, Wijers OB, Lagerwaard FJ, Levendag PC. A three-dimensional CT-based target definition for elective irradiation of the neck. Int J Radiat Oncol Biol Phys 1999;45(1):33-9.

51. Fu KK, Pajak TF, Trotti A, Jones CU, Spencer SA, Phillips TL, et al. A Radiation Therapy Oncology Group (RTOG) phase III randomized study to compare hyperfractionation and two variants of accelerated fractionation to standard fractionation radiotherapy for head and neck squamous cell carcinomas: first report of RTOG 9003. Int J Radiat Oncol Biol Phys 2000;48(1):7-16.

52. Lee N, Xia P, Quivey JM, Sultanem K, Poon I, Akazawa C, et al. Intensity-modulated radiotherapy in the treatment of nasopharyngeal carcinoma: an update of the UCSF experience. Int J Radiat Oncol Biol Phys 2002;53(1):12-22.

53. Xia P, Fu KK, Wong GW, Akazawa C, Verhey U. Comparison of treatment plans involving intensity-modulated radiotherapy for nasopharyngeal carcinoma. Int J Radiat Oncol Biol Phys 2000;48(2):329-37.

54. Sultanem K, Shu HK, Xia P, Akazawa C, Quivey JM, Verhey $\amalg$, et al. Three-dimensional intensity-modulated radiotherapy in the treatment of nasopharyngeal carcinoma: the University of California-San Francisco experience. Int J Radiat Oncol Biol Phys 2000;48(3):711-22.

55. Eisbruch A, Kim HM, Terrell JE, Marsh LH, Dawson LA, Ship JA. Xerostomia and its predictors following parotidsparing irradiation of head-and-neck cancer. Int J Radiat Oncol Biol Phys 2001;50(3):695-704. 